\title{
Orthogonality of Case's Eigenfunctions in One-Speed Transport Theory*
}

\author{
I. Kuščer, $\dagger$ N. J. MCCormick, and G. C. Summerfield \\ Department of Nuclear Engineering, The University of Michigan, Ann Arbor, Michigan
}

\begin{abstract}
The eigenfunctions of the one-speed transport equation, as introduced by Case, are shown to have more general orthogonality properties than previously known. In particular, for isotropic scattering, partial range and "two-media" orthogonality relations are derived. An extension lo linearly anisotropic scattering is indicated. These results facilitate the application of Case's method to one-speed transport problems in plane geometry.
\end{abstract}

\section{INTRODUCTION}

Several problems in one-speed neutron transport theory have been solved (1-6) by a method developed by Case (1). This method involves the expansion of the angular density in terms of the eigenfunctions of the homogeneous transport equation. Furthermore, Case has derived full-range orthogonality relations that greatly facilitate the determination of the expansion coefficients for infinite medium problems. However, for other problems, one had to use the more tedious constructive method (1).

It will be shown that the orthogonality relations can be generalized to any partial range (Section II), and especially to a half range (Section III). Also, "two-media" orthogonality relations for isotropic scattering will be derived (Section IV). The generalization to anisotropic scattering requires the introduction of bi-orthogonality relations, as indicated in Section V. With these orthogonality relations, half-space and two half-space problems can be solved almost as easily as infinite medium problems.

First, let us review some of Case's results. For isotropic scattering and plane symmetry, the homogeneous one-speed transport equation is

$$
\mu \frac{\partial \psi(x, \mu)}{\partial x}+\psi(x, \mu)=\frac{c}{2} \int_{-1}^{1} \psi\left(x, \mu^{\prime}\right) d \mu^{\prime} .
$$

* Work supported in part by the United States Atomic Energy Commission.

$\dagger$ Fulbright-Hays visiting seholar, on leave of absence from The University of Ljubljana, Yugoslavia. 
Its eigenfunctions are

$$
\phi_{\nu}(\mu) e^{-x / \nu}
$$

and

$$
\phi_{ \pm}(\mu) e^{\mp x / \nu_{0}}
$$

where

$$
\begin{gathered}
\phi_{\nu}(\mu)=\frac{c \nu}{2} P \frac{1}{\nu-\mu}+\lambda(\nu) \delta(\nu-\mu), \quad-1 \leqq \nu \leqq 1, \\
\phi_{ \pm}(\mu)=\frac{c \nu_{0}}{2} \frac{1}{\nu_{0} \mp \mu}, \\
\lambda(\nu)=1-c \nu \tanh ^{-1} \nu,
\end{gathered}
$$

and $\nu_{0}$ is defined by

$$
\Lambda\left( \pm \nu_{0}\right)=0
$$

where

$$
\Lambda(z)=1-c z \tanh ^{-1}(1 / z) .
$$

The limiting values of $\Lambda(\nu)$ on the cut, $-1<\nu<1$, are

$$
\Lambda^{ \pm}(\nu)=\lambda(\nu) \pm 1 / 2 i \pi c \nu .
$$

The symbol $P$ in Eq. (2c) is a reminder that we must take the Cauchy principal value of integrals over $\mu$ or $\nu$.

Case has shown that an arbitrary function $\psi(\mu)$, defined on the interval $\alpha \leqq \mu \leqq \beta$, where $-1 \leqq \alpha<\beta \leqq 1$, can be expanded as

$$
\psi(\mu)=a_{+} \phi_{+}(\mu)+a_{-} \phi_{-}(\mu)+\int_{\alpha}^{\beta} A(\nu) \phi_{\nu}(\mu) d \nu,
$$

where one can take $a_{+}=0$ unless $\beta=1, \alpha \leqq 0$, and $a_{-}=0$ unless $\alpha=-1$, $\beta \geqq 0$. For $\alpha=-1, \beta=1$, Case derived the following very useful orthogonality relations:

$$
\begin{aligned}
& \int_{-1}^{1} \phi_{\nu}(\mu) \phi_{\nu^{\prime}}(\mu) \mu d \mu=\nu \Lambda^{+}(\nu) \Lambda^{-}(\nu) \delta\left(\nu-\nu^{\prime}\right), \\
& \int_{-1}^{1} \phi_{\nu}(\mu) \phi_{ \pm}(\mu) \mu d \mu=0 \\
& \int_{-1}^{1} \phi_{+}(\mu) \phi_{-}(\mu) \mu d \mu=0 .
\end{aligned}
$$


The integral in Eq. (6a) requires some discussion since part of it, i.e.,

$$
\frac{c^{2} \nu \nu^{\prime}}{4} \int_{-1}^{1} P \frac{1}{\nu-\mu} P \frac{1}{\nu^{\prime}-\mu} \mu d \mu
$$

does not have a unique meaning when $\nu \rightarrow \nu^{\prime}$. Using the definitions

$$
\begin{aligned}
P \frac{1}{x} & =\lim _{\epsilon \rightarrow 0} \frac{x}{x^{2}+\epsilon^{2}}, \\
\pi \delta(x) & =\lim _{\epsilon \rightarrow 0^{+}} \frac{\epsilon}{x^{2}+\epsilon^{2}},
\end{aligned}
$$

where the limit $\epsilon \rightarrow 0$ is to be taken only after the integration over $x$ is performed we understand the singular factor of the integrand in Eq. (7) as

$$
\begin{aligned}
P \frac{1}{\nu-\mu} P \frac{1}{\nu^{\prime}-\mu}=\frac{1}{\nu-\nu^{\prime}}\left(P \frac{1}{\nu^{\prime}-\mu}-P \frac{1}{\nu-\mu}\right) & \\
& +\pi^{2} \delta(\nu-\mu) \delta\left(\nu^{\prime}-\mu\right) .
\end{aligned}
$$

This has been used in Eq. (6a), and shall be used for all integrals of a similar type.

Equation (9) is a restatement of the Poincaré-Bertrand formula $(1,7)$, and has the advantage of allowing the formal inversion of the order of integration in cases such as the following:

$$
\int \phi_{\nu^{\prime}}(\mu) d \mu \int A(\nu) \phi_{\nu}(\mu) d \nu=\int A(\nu) d \nu \int \phi_{\nu^{\prime}}(\mu) \phi_{\nu}(\mu) d \mu .
$$

\section{GENERAL-RANGE ORTHOGONALITY}

We shall first derive the orthogonality relations for a general range, $\alpha \leqq \nu \leqq \beta$, $-1 \leqq \alpha<\beta \leqq 1$. This treatment, although too broad for known application in neutron transport theory, will display the generality of the technique.

Consider (1)

$$
X_{0}(z)=\exp \left\{\frac{1}{2 \pi i} \int_{\alpha}^{\beta} \ln \left(\frac{\Lambda^{+}(\nu)}{\Lambda^{-}(\nu)}\right) \frac{d \nu}{\nu-z}\right\} .
$$

This function is analytic in the complex $z$-plane (including infinity), cut along $\alpha<z<\beta$, and satisfies the ratio condition,

$$
\frac{X_{0}^{+}(\nu)}{\bar{X}_{0}^{-}(\nu)}=\frac{\Lambda^{+}(\nu)}{\Lambda^{-}(\nu)}, \quad \alpha<\nu<\beta,
$$

the Hölder condition, and the conditions 


$$
\begin{aligned}
& \left|X_{0}(z)\right|=O\left(|z-\alpha|^{\kappa}\right), \quad z \rightarrow \alpha, \\
& \left|X_{0}(z)\right|=O\left(|z-\beta|^{\kappa}\right), \quad z \rightarrow \beta,
\end{aligned}
$$

where $\kappa>-1$.

All these conditions are also satisfied by

$$
\frac{X_{0}(z)}{z-\alpha}, \quad \text { if } \quad \alpha<0,
$$

and

$$
\frac{X_{0}(z)}{\beta-z}, \quad \text { if } \beta>0,
$$

and by any linear combination of (11), (14a), and (14b) if we exclude (14a) whenever $\alpha \geqq 0$ and (14b) whenever $\beta \leqq 0$. For any such function, we shall use the notation $X(z)$. The behaviour of the $X(z)$ for large $z$ can be inferred from

$$
X_{0}(\infty)=1 \text {. }
$$

Now, we define

$$
\gamma(\mu)=\frac{c \mu}{2} \frac{X^{+}(\mu)}{\Lambda^{+}(\mu)}, \quad \alpha \leqq \mu \leqq \beta .
$$

This can also be expressed as

$$
\gamma(\mu)=(1 / 2 \pi i)\left[X^{+}(\mu)-X^{-}(\mu)\right],
$$

whereas

$$
\gamma(\mu) \lambda(\mu)=1 / 4 c \mu\left[X^{+}(\mu)+X(\mu)\right] .
$$

Clearly, we can have up to three linearly independent $\gamma(\mu)$. We denote one of them as

$$
\gamma_{0}(\mu)=\frac{c \mu}{2} \frac{X_{0}{ }^{+}(\mu)}{\Lambda^{+}(\mu)}
$$

Applying Cauchy's integral theorem to $X(z)$, and using Eq. (15b), one can show that (1)

$$
\int_{\alpha}^{\beta} \gamma(\mu) \frac{d \mu}{\mu-z}=X(z)-X(\infty) .
$$

For $z=\nu, \alpha \leqq \nu \leqq \beta$, this equation gives

$$
\frac{c \nu}{2} P \int_{\alpha}^{\beta} \gamma(\mu) \frac{d \mu}{\nu-\mu}=\frac{c \nu}{2} X(\infty)-\gamma(\nu) \lambda(\nu),
$$

where we have used Eq. (16). 
Equation (19) can be rewritten as

$$
\int_{\alpha}^{\beta} \phi_{\nu}(\mu) \gamma(\mu) d \mu=\frac{1}{2} c \nu X(\infty), \quad \alpha \leqq \nu \leqq \beta,
$$

or also as

$$
X(\infty)(\nu-\mu) \phi_{\nu}(\mu)=\int_{\alpha}^{\beta} \phi_{\nu}\left(\mu^{\prime}\right) \gamma\left(\mu^{\prime}\right) d \mu^{\prime}, \quad \alpha \leqq \nu \leqq \beta .
$$

We then proceed in the usual way, i.e., we write this equation twice, for $\phi_{\nu}(\mu)$ and $\phi_{\nu^{\prime}}(\mu)$, multiply by $\phi_{\nu^{\prime}}(\mu) \gamma(\mu) d \mu$ and $\phi_{\nu}(\mu) \gamma(\mu) d \mu$, respectively, integrate, and subtract. The result shows that the $\phi_{\nu}(\mu), \alpha \leqq \nu \leqq \beta$ are mutually orthogonal in the interval $\alpha \leqq \mu \leqq \beta$ with respect to the weight function $\gamma(\mu)$. By applying the partial fraction analysis, as explained in Section I, we evaluate also the normalization factor:

$$
\begin{aligned}
\int_{\alpha}^{\beta} \phi_{\nu}(\mu) \phi_{\nu^{\prime}}(\mu) \gamma(\mu) d \mu=\gamma(\nu) \Lambda^{+}(\nu) \Lambda^{-}(\nu) \delta\left(\nu-\nu^{\prime}\right), & \\
\alpha & \leqq \nu \leqq \beta, \alpha \leqq \nu^{\prime} \leqq \beta .
\end{aligned}
$$

This relation can be used to determine the coefficients $A(v)$ in an expansion such as $(\tilde{j})$.

Since the weight function $\gamma(\nu)$ is still somewhat arbitrary, we can select a particular one which meets certain additional requirements. For example, if $0 \leqq \alpha<\beta \leqq 1$, there are two linearly independent $\gamma(\mu)$; and if we want $\phi_{+}(\mu)$ to be included in the orthogonality relations, we must take

$$
\gamma(\mu)=\frac{\nu_{0}-\mu}{\beta-\mu} \gamma_{0}(\mu)
$$

as we can see from Eq. (20).

If we have three linearly independent $\gamma(\mu)$, that is, if $\alpha<0, \beta>0$, then the choice,

$$
\gamma(\mu)=\frac{\nu_{0}^{2}-\mu^{2}}{(\mu-\alpha)(\beta-\mu)} \gamma_{0}(\mu),
$$

provides full orthogonality of the $\phi_{\nu}(\mu), \alpha \leqq \nu \leqq \beta, \phi_{+}(\mu)$ and $\phi_{-}(\mu)$.

We shall not pursue the general range formalism any further but turn now to the two special cases of interest in transport theory.

In the full range case $(\alpha=-1, \beta=1)$, the ratio $X(z) / \Lambda(z)$ reduces to a rational function (1), and the weight function (24) is simply

$$
\frac{\nu_{0}^{2}-\mu^{2}}{1-\mu^{2}} \gamma_{0}(\mu)=\frac{c}{2(1-c)} \mu
$$

in agreement with Eqs. $(6 a-c)$. 
The analogous specialization to the half range will be carried out in the next section.

\section{HALF-RANGE ORTHOGONALITY}

In this case ( $\alpha=0, \beta=1)$, it is convenient to make a slight change in notation by introducing the following particular $X$-function and $\gamma$-function (1):

$$
\begin{gathered}
X(\mu)=X_{0}(\mu) /(1-\mu), \\
\gamma(\mu)=1 / 2 c \mu X^{+}(\mu) / \Lambda^{+}(\mu)=\gamma_{0}(\mu) /(1-\mu) .
\end{gathered}
$$

Sometimes the moments of this $\gamma(\mu)$ are useful:

$$
\begin{aligned}
\gamma^{(n)} & =\int_{0}^{1} \mu^{n} \gamma(\mu) d \mu, \\
\gamma^{(0)} & =1 .
\end{aligned}
$$

The weight function (23) needed for the full orthogonality of $\phi_{+}(\mu)$ and $\phi_{\nu}(\mu)$, $0 \leqq \nu \leqq 1$, can now be written as

$$
\left(\nu_{0}-\mu\right) \gamma(\mu) \text {. }
$$

The corresponding formulas are given in the summary at the end of this section.

As an example of the use of these formulas, consider the half-space albedo problem (1). That is, consider Eq. (1), with $x \geqq 0$, and the boundary conditions

$$
\psi(0, \mu)=\delta\left(\mu-\mu_{0}\right), \quad \mu>0,
$$

where $\mu_{0}>0$, and

$$
\lim _{x \rightarrow \infty} \psi(x, \mu)=0 .
$$

In view of Eq. (30b), the solution is sought in the form

$$
\psi(x, \mu)=a_{+} \phi_{+}(\mu) e^{-x / \nu_{0}}+\int_{0}^{1} A(\nu) \phi_{\nu}(\mu) e^{-x / \nu} d \nu,
$$

so that Eq. (30a) gives

$$
\delta\left(\mu-\mu_{0}\right)=a_{+\phi_{+}}(\mu)+\int_{0}^{1} A(\nu)_{\phi_{\nu}}(\mu) d \nu .
$$

From this, we can immediately write the expressions for the expansion coefficients, using (A.1, 2, 4), and relying on Case's completeness theorem,

$$
\begin{aligned}
a_{+} & =-\frac{2 \gamma\left(\mu_{0}\right)}{c \nu_{0} X\left(\nu_{0}\right)}, \\
A(\nu) & =\frac{\left(\nu_{0}-\mu_{0}\right) \gamma\left(\mu_{0}\right) \phi_{\nu}\left(\mu_{0}\right)}{\left(\nu_{0}-\nu\right) \gamma(\nu) \Lambda^{+}(\nu) \Lambda^{-}(\nu)} .
\end{aligned}
$$


For $x=0$ the integration in (31), with (34) substituted, can actually be carried out (1), by applying Cauchy's integral theorem to the function $\left[\left(\nu_{0}-z\right)\left(z-\mu_{0}\right) X(z)\right]^{-1}$. This leads to the known expression for the reflected angular density.

Similarly Milne's problem (1), the problem with a uniform source distribution, and some other half-space problems can be solved, using the orthogonality relations. These also help to shorten the initial step in the solution of slab problems $(3,6)$, namely the conversion of the singular integral equation for the expansion coefficients into an equation of the Fredholm type.

\section{Summary of Half-Range Formulas}

In all the formulas, $0 \leqq \nu \leqq 1,0 \leqq \nu^{\prime} \leqq 1$.

$$
\begin{aligned}
\int_{\mathfrak{s}}^{1} \phi_{\nu}(\mu) \phi_{\nu^{\prime}}(\mu)\left(\nu_{0}-\mu\right) \gamma(\mu) d \mu & =\left(\nu_{0}-\nu\right) \gamma(\nu) \Lambda^{+}(\nu) \Lambda^{-}(\nu) \delta\left(\nu-\nu^{\prime}\right) \\
\int_{0}^{1} \phi_{+}(\mu) \phi_{\nu}(\mu)\left(\nu_{0}-\mu\right) \gamma(\mu) d \mu & =0 \\
\int_{0}^{1} \phi_{-}(\mu) \phi_{\nu}(\mu)\left(\nu_{0}-\mu\right) \gamma(\mu) d \mu & =c \nu \nu_{0} X\left(-\nu_{0}\right) \phi_{-}(\nu) \\
\int_{0}^{1} \phi_{ \pm}(\mu) \phi_{+}(\mu)\left(\nu_{0}-\mu\right) \gamma(\mu) d \mu & =\mp\left(1 / 2 c \nu_{0}\right)^{2} X\left( \pm \nu_{0}\right) \\
\int_{0}^{1} \phi_{-\nu}(\mu) \phi_{+}(\mu)\left(\nu_{0}-\mu\right) \gamma(\mu) d \mu & =1 / 4 c^{2} \nu \nu_{0} X(-\nu) \\
\int_{0}^{1} \phi_{-\nu}(\mu) \phi_{\nu^{\prime}}(\mu)\left(\nu_{0}-\mu\right) \gamma(\mu) d \mu & =1 / 2 c \nu^{\prime} \phi_{-\nu}\left(\nu^{\prime}\right)\left(\nu_{0}+\nu\right) X(-\nu) \\
\int_{0}^{1} \phi_{\nu}(\mu)\left(\nu_{0}-\mu\right) \gamma(\mu) d \mu & =1 / 2 c \nu \\
\int_{0}^{1} \mu \phi_{\nu}(\mu)\left(\nu_{0}-\mu\right) \gamma(\mu) d \mu & =1 / 2 c \nu\left[\gamma^{(1)}-\left(\nu_{0}-\nu\right)\right]
\end{aligned}
$$

Remark: Formulas for a medium with no absorption are obtained if the above equations are divided by $\nu_{0}$ on both sides and then the limit $c \rightarrow 1$ (which entails $\nu_{0} \rightarrow \infty$ ) carried out.

\section{IV. "TWO-MEDIA" ORTHOGONALITY}

Another case of interest is a system with plane symmetry and two adjoining media which differ in the value of $c$, say $c=c_{1}$ on the right-hand side of the interface and $c=c_{2}$ on the left-hand side. Accordingly, we must distinguish $\nu_{01}$ and $\nu_{v 2}, \phi_{1 \pm}(\mu)$ and $\phi_{2 \pm}(\mu), \phi_{1 v}(\mu)$ and $\phi_{2 v}(\mu), X_{1}(z)$ and $X_{2}(z)$, and similarly for the 
other relevant functions, each defined as in Section III, with the appropriate value $c_{1}$ or $c_{2}$.

It is convenient to introduce the following notation $(1,5)$ :

$$
\begin{gathered}
c(\nu)= \begin{cases}c_{1}, & \nu>0, \\
c_{2}, & \nu<0,\end{cases} \\
\Phi_{\nu}(\mu)=\frac{\nu c(\nu)}{2} P \frac{1}{\nu-\mu}+l(\nu) \delta(\nu-\mu), \\
l(\nu)=1-\nu c(\nu) \tanh ^{-1} \nu, \\
L^{ \pm}(\nu)=l(\nu) \pm \frac{1}{2} i \pi \nu c(\nu), \\
\chi(z)=X_{1}(z) X_{2}(-z), \\
\Gamma(\mu)=1 / 2 \mu c(\mu) \chi^{+}(\mu) / L^{+}(\mu) .
\end{gathered}
$$

It can be shown $(1,5)$ that:

$$
\begin{aligned}
& \int_{-1}^{1} \mu^{n} \Gamma(\mu) \frac{d \mu}{\mu-z}=z^{n} \chi(z), \quad n=0,1, \\
& \int_{-1}^{1} \mu^{2} \Gamma(\mu) \frac{d \mu}{\mu-z}=z^{2} \chi(z)+1 .
\end{aligned}
$$

These equations lead to:

$$
\begin{gathered}
\int_{-1}^{1} \mu^{n} \Gamma(\mu) \Phi_{\nu}(\mu) \Phi_{\nu^{\prime}}(\mu) d \mu=\nu^{n} \Gamma(\nu) L^{+}(\nu) L^{-}(\nu) \delta\left(\nu-\nu^{\prime}\right), \quad n=0,1,2, \\
\int_{-1}^{1} \mu^{n} \Gamma(\mu) \Phi_{\nu}(\mu) d \mu=0, \quad n=0,1 .
\end{gathered}
$$

The three linearly independent weight functions $\mu^{n} \Gamma(\mu), n=0,1,2$, can be combined such that the orthogonality relations include any two of the four discrete eigenfunctions, $\phi_{1,2 \pm}(\mu)$. We shall include $\phi_{1+}(\mu)$ and $\phi_{2-}(\mu)$ by taking the following weight function:

$$
\left(\nu_{01}-\mu\right)\left(\nu_{02}+\mu\right) \Gamma(\mu) .
$$

The orthogonality relations with this weight function and some related formulas are summarized at the end of this section. These formulas facilitate the solution of typical two-media problems, like those considered by Mendelson and Summerfield $(5)$.

The results of this section embody as special cases both the half-range and full-range results. The latter can be seen by observing that, for $c_{1}=c_{2}=c$, we have $\chi(z)=X(z) X(-z)=\Lambda(z) /(1-c)\left(\nu_{0}^{2}-z^{2}\right)$. Thus the weight function 
reduces to:

$$
\left(\nu_{0}^{2}-\mu^{2}\right) \Gamma(\mu)=\frac{c}{2(1-c)} \mu
$$

in accord with Eqs. (6a-c).

For $c_{2}=0$, we have $\nu_{02}=1, X_{2}(z)=1 /(1-z)$, and therefore $\chi(z)=X_{1}(z) /$ $(1+z)$. Thus the weight function for this case reduces to:

$$
\left(\nu_{01}-\mu\right)(1+\mu) \Gamma(\mu)=\left\{\begin{array}{l}
\frac{1}{2} 2 c \mu\left(\nu_{01}-\mu\right) X_{1}^{+}(\mu) / \Lambda_{1}^{+}(\mu), \quad 0<\mu \leqq 1, \\
0, \quad-1 \leqq \mu<0,
\end{array}\right.
$$

in agreement with Eqs. (A.1, 2).

The partial range case can be included in this scheme if $c(\nu)$ is taken to be cons tant for $\alpha \leqq \nu \leqq \beta$ and zero otherwise. Instead of $\chi(z)$, as defined by Fq. (39), we"then"need

$$
\chi_{0}(z)=\exp \left\{\frac{1}{2 \pi i} \int_{-1}^{1} \ln \frac{L^{+}(\nu)}{L^{-}(\nu)} \frac{d \nu}{\nu-z}\right\} .
$$

This suggests an even broader generalization by taking $c(\nu)$ to be an arbitrary function of $\nu$. However, we cannot now see any application for such a generalization.

Summary of "Two-Media" Formulas

$$
\begin{aligned}
& \int_{-1}^{1} \Phi_{\nu}(\mu) \Phi_{\nu^{\prime}}(\mu)\left(\nu_{01}-\mu\right)\left(\nu_{02}+\mu\right) \Gamma(\mu) d \mu \\
& =\left(\nu_{01}-\nu\right)\left(\nu_{02}+\nu\right) \Gamma(\nu) L^{+}(\nu) L^{-}(\nu) \delta\left(\nu-\nu^{\prime}\right) \\
& \int_{-1}^{1} \phi_{1+}(\mu) \Phi_{\nu}(\mu)\left(\nu_{01}-\mu\right)\left(\nu_{02}+\mu\right) \Gamma(\mu) d \mu=0 \\
& \int_{-1}^{1} \phi_{2-}(\mu) \Phi_{\nu}(\mu)\left(\nu_{01}-\mu\right)\left(\nu_{02}+\mu\right) \Gamma(\mu) d \mu=0 \\
& \int_{-1}^{1} \phi_{1}+(\mu) \phi_{2}-(\mu)\left(\nu_{01}-\mu\right)\left(\nu_{02}+\mu\right) \Gamma(\mu) d \mu=0 \\
& \int_{-1}^{1} \phi_{1-}(\mu) \Phi \nu(\mu)\left(\nu_{01}-\mu\right)\left(\nu_{02}+\mu\right) \Gamma(\mu) d \mu \\
& =\nu c(\nu) \nu_{01}\left(\nu_{02}-\nu_{01}\right) \chi\left(-\nu_{01}\right) \phi_{1-}(\nu) \\
& \int_{-1}^{1} \phi_{2+}(\mu) \Phi_{\nu}(\mu)\left(\nu_{01}-\mu\right)\left(\nu_{02}+\mu\right) \Gamma(\mu) d \mu \\
& =\nu c(\nu)_{\nu_{02}}\left(\nu_{01}-\nu_{02}\right) \chi\left(\nu_{02}\right) \phi_{2+}(\nu)
\end{aligned}
$$




$$
\begin{aligned}
\int_{-1}^{1} \phi_{1 \pm}(\mu)_{\phi_{1}+}(\mu)\left(\nu_{01}-\mu\right) & \left(\nu_{02}+\mu\right) \Gamma(\mu) d \mu \\
= & -\left(1 / 2 c_{1} \nu_{01}\right)^{2}\left(\nu_{01} \pm \nu_{02}\right) \chi\left( \pm \nu_{01}\right) \\
\int_{-1}^{1} \phi_{2 \pm}(\mu) \phi_{2-}(\mu)\left(\nu_{01}-\mu\right) & \left(\nu_{02}+\mu\right) \Gamma(\mu) d \mu \\
& =\left(1 / 2 c_{2} \nu_{02}\right)^{2}\left(\nu_{02} \pm \nu_{01}\right) \chi\left( \pm \nu_{02}\right) \\
& =-1 / 2 c_{1} c_{2} \nu_{01} \nu_{02}^{2} \chi\left(\nu_{02}\right) \\
\int_{-1}^{1} \phi_{2+}(\mu)_{\phi_{1+}}(\mu)\left(\nu_{01}-\mu\right) & \left(\nu_{02}+\mu\right) \Gamma(\mu) d \mu \\
\int_{-1}^{1} \phi_{1-}(\mu) \phi_{2-}(\mu)\left(\nu_{01}-\mu\right) & \left(\nu_{02}+\mu\right) \Gamma(\mu) d \mu \\
= & 1 / 2 c_{1} c_{2} \nu_{01}^{2} \nu_{02} \chi\left(-\nu_{01}\right) .
\end{aligned}
$$

Further integrals, which involve $\phi_{1 \nu}(\mu)$ for $\nu<0$ and $\phi_{2 \nu}(\mu)$ for $\nu>0$, occur in multislab problems. However, these integrals can be reduced to (B.1-3) by observing the identity

$$
\begin{gathered}
c_{2} \phi_{1 \nu}(\mu)-c_{1} \phi_{2 \nu}(\mu)=\left(c_{2}-c_{1}\right) \delta(\nu-\mu) . \\
\text { V. OUTLOOK }
\end{gathered}
$$

When considering anisotropic scattering, it turns out that this generalization is not quite straightforward, except in the full-range case, where relations of the form $(6 \mathrm{a}-\mathrm{c})$ are still valid $(\mathscr{Q})$. It appears that for the half-range no full orthogonality relation is available which would involve all the eigenfunctions needed in the expansions. Instead, one must resort to bi-orthogonality reiations.

In the half-range case with linearly anisotropic scattering, the definitions and identities of Shure and Natelson (4) lead to the relation

$$
\begin{aligned}
\int_{0}^{1} \phi_{\nu}(\mu)\left[\phi_{\nu^{\prime}}(\mu)+B \frac{c \nu^{\prime}}{2}\right]\left(\nu_{0}-\mu\right) \gamma(\mu) d \mu \\
=\left(\nu_{0}-\nu\right) \gamma(\nu) \Lambda^{+}(\nu) \Lambda^{-}(\nu) \delta\left(\nu-\nu^{\prime}\right),
\end{aligned}
$$

and to similar formulas involving $\phi_{ \pm}(\mu)$, with

$$
B=b_{1}(1-c)\left(\nu_{0}-\bar{\nu}\right) / d\left(\nu_{0} \bar{\nu}\right),
$$

and with the other symbols having the same meaning as in ref. 4. By application of these formulas, simple half-space problems can be solved just as easily as for isotropic seattering.

More details about this and about other cases of anisotropic scattering will be given elsewhere (8). 
The various generalizations of the completeness theorem and the orthogonality relations leave little doubt that we are dealing here with general properties of a certain class of linear integrodifferential equations. Therefore, rather than pushing ahead by stepwise generalizations to higher order anisotropic scattering and the like, one is tempted to ask what is that class of equations.

Perhaps a closer link to broader theorems in the theory of linear functional equations could be established. One might ask for expansions applicable to arbitrary functions $f(x, \mu)$, where both $c$ and $\nu$ would appear as running parameters. Expansions of this type have already been encountered in time-dependent transport problems $(9,10)$.

The answers to such questions might provide a better understanding of the mathematical background of Case's method.

\section{ACKNOWLEDGMENTS}

We wish to thank Professor P. F. Zweifel for stimulating suggestions and encouragement. One of us (I. K.) wishes to express his gratitude to the members of the Department of Nuclear Engineering, The University of Michigan, for the hospitality shown him.

Received: May 7, 1964

\section{REFERENCES}

1. K. M. CASE, Ann. Phys. (N.Y.) 9, 1 (1960); and "Recent Developments in Neutron Transport Theory." Michigan Memorial Phoenix Project, The University of Michigan, 1961.

2. J. R. Mika, Nucl. Sci. Eng. 11, 415 (1961).

3. G. J. Mitsis, ANL-6459; Nucl. Sei. Eng. 17, 55 (1963).

4. F. C. Shure and M. Natelson, Ann. Phys. (N.Y.) 26, 279 (1964).

5. M. R. Mendelson and G. C. Summerfield, J. Math. Phys. 5, 668 (1964).

6. N. J. McCormick and M. R. Mendelson, Nucl. Sci. Eng., in press.

7. N. Muskhelishvili, "Singular Integral Equations." Noordhoff, Groningen, Holland, 1953.

8. N. J. McConmick, Thesis, University of Michigan (1964).

9. R. L. Bowden, Thesis, Virginia Polytechnic Institute (1963); also Report TID 18884 (1963).

10. I. Kuščer ANd P. F. ZWEIFEL, J. Math. Phys., in press. 